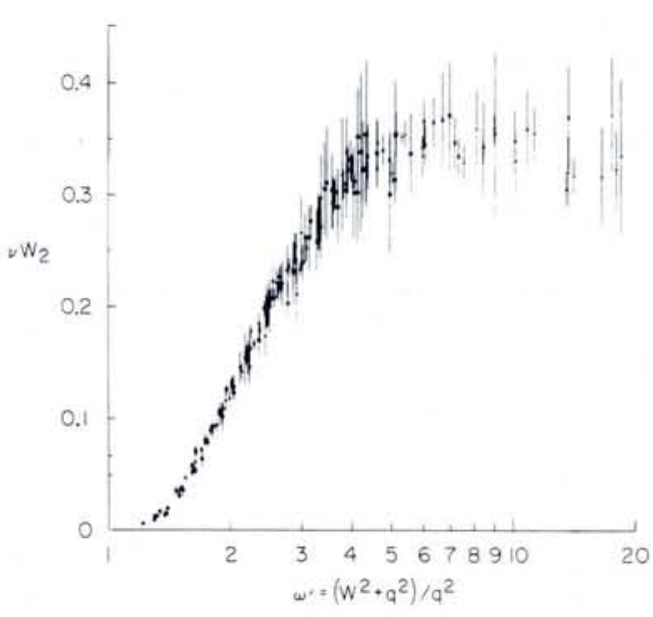

"scaling" (Fig. 2), is what is expected for scattering on point-like constituents within the target proton. The 1990 Nobel Prize has been awarded for this remarkably clear result from a difficult experiment.

J.D. Björken, a theoretician at SLAC, had in fact predicted in 1967 the scaling behaviour long before the results of the SLAC-MIT experiment were available.
Fig. 2 - The scaling behaviour of deep inelastic scattering of electrons on protons. Data from the SLAC-MIT experiment showing that the structure function $W_{2}$ where $v$ is the transferred energy, depends only on the ratio $2 \mathrm{mv} / \mathrm{q}^{2}$ where $m$ is the proton mass and $q$ is the transferred momentum. This behaviour is expected if scattering occurs on point-like constituents in the target nucleons. From Miller G. et al., Phys. Rev. D5 (1972) 528 with permission.

He used a tool called Current Algebra which had been developed as a theoretical device for attaining wide-ranging properties. The quark theory that could be used was considered too elementary to be correct and it invoked quark fields for which no particles were known. Yet there were strong reasons to believe that the Current Algebra relations which could be thus derived were far more general than the theory within which they were obtained.

The quark structure of matter itself dated back to the early sixties and the success of the "eightfold way" of M. Gell-Mann and Y. Ne'eman. Large numbers of particles called hadrons, which resembled nucleons or $\pi$-mesons in some respects, had been discovered. The similarities and differences among these hadrons could be accounted for in terms of Unitary Symmetry, and the simplest visualization of the abstract mathematical description became much more comprehensible when $\mathrm{M}$. Gell-Mann and G. Zweig proposed that hadrons could be "constructed" in terms of building blocks called quarks for the baryons, and a quark-antiquark pair for the mesons The properties of these hypothetical particles, and in particular their fractional charges, were however such that few believers came forward. Quarks, moreover, had been sought but never found.

Whilst Björken's approach was carefully phrased in terms of the properties
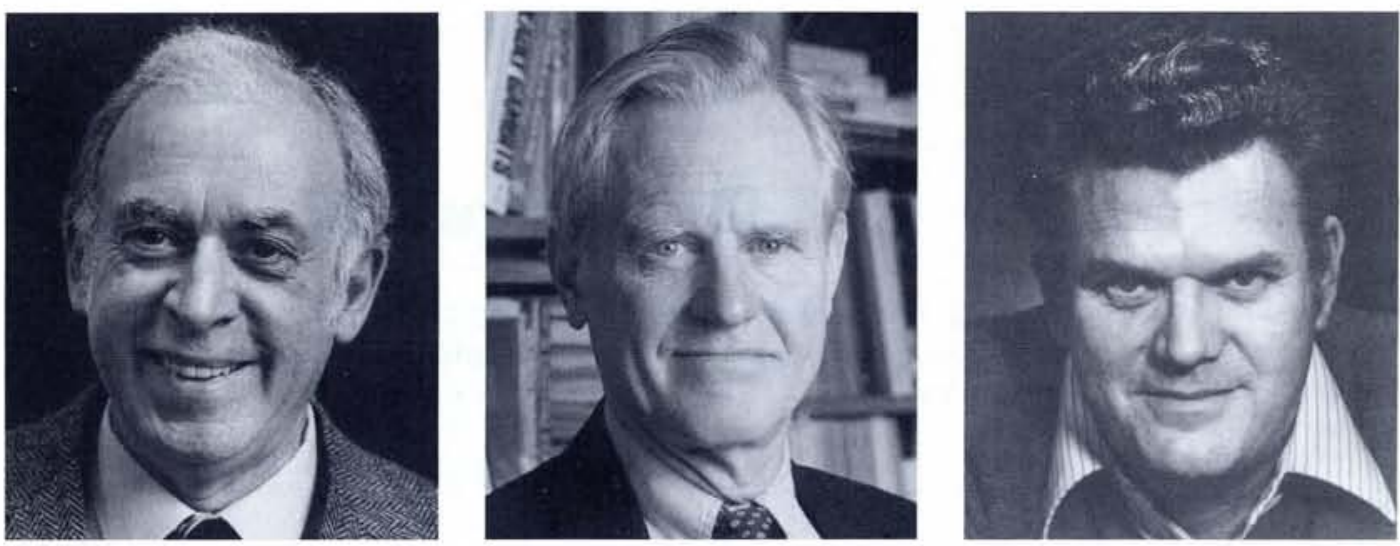

The 1990 Nobel laureates: Jerome Friedman, Henry Kendall and Richard Taylor (from left to right).

Jerome I. Friedman was named last year as the William A. Coolidge Professor of Physics in the Laboratory for Nuclear Science (LNS), Massachusetts Institute of Technology, Cambridge, MA, USA. Professor Friedman was born in 1930 in Chicago and graduated from the University of Chicago where he stayed for one year before becoming a Research Associate at Stanford University in 1957, and an Associate Professor at MIT in 1960. Apart from his contributions to the SLAC-MIT experiment, he is also well known as the co-discoverer of the violation of parity conservation in the decay of the $\pi$-meson. Awarded the W.K.H. Panofsky Prize jointly with the other Laureates in 1989, Professor Friedman is a Fellow of the American Physical Society and a Member of the American Academy of Arts and Sciences. He has been the Director of the LNS (1980-83) and Head of MIT's Physics Department (1983-88), and he is currently serving on the Scientific Policy Committee of the Superconducting Super Collider Laboratory, TX, USA.

\title{
The 1990 Nobel Laureates
}

Henry W. Kendall has been a Professor of Physics in MIT's Laboratory for Nuclear Science since 1967. Born in Boston in 1926, he graduated from MIT in 1955 and then worked as a National Science Foundation postdoc at the Brookhaven National Laboratory and MIT for three years. Following six years in the Physics Department, Stanford University, he joined the MIT faculty in 1961. He is a founding member of the Union of Concerned Scientists (UCS) and in this capacity has served on various panels dealing with reactor safety. $\mathrm{He}$ has been the Chairman of the UCS Board of Directors since 1973 and a member of the Board of Directors of the Arms Control Association since 1979 . He is also a Fellow of the APS and the AAAS and received the Leo Szilard Award and the Bertrand Russell Society Award in 1982
Richard E. Taylor is Professor of Physics at the Stanford Linear Accelerator Center (SLAC), Menlo Park, CA, USA. Born in Alberta, Canada in 1929, he graduated with an M.Sc. from the University of Alberta in 1952 and with a Ph.D. from Stanford University in 1962. After holding research positions at both the Laboratoire de I'Accélérateur Linéaire, Orsay, France and the Lawrence Radiation Laboratory, Berkeley, CA, USA, he joined the SLAC staff, becoming Professor in 1968 and serving as the Associate Director for Research from $1982-86$. He is involved in the preparation of one the HERA experiments at DESY, Hamburg which will move into the beam next August, and he served on the LEP Experiments Committee at CERN, Geneva until last year. He is a Fellow of the APS and of the Royal Society of Canada. 